\title{
Trends in Ambulatory Electronic Consultations During the COVID-19 Pandemic
}

J Gen Intern Med 35(10):3117-9

DOI: $10.1007 / \mathrm{s} 11606-020-05878-\mathrm{Z}$

(c) Society of General Internal Medicine 2020

\section{INTRODUCTION}

SARS-CoV2, the virus responsible for the COVID-19 pandemic, ${ }^{1}$ has forced rapid changes in healthcare delivery. Telehealth has previously played a role in delivering ambulatory care in the setting of similar disasters. ${ }^{2}$ Electronic consultations (e-consults), in particular, may be an effective method of sustaining specialty consultative care while preserving social distancing and reducing demands for personal protective equipment. Hypothesizing that e-consult requests would increase during the pandemic, we sought to define COVID-19associated changes in e-consult requests.

\section{METHODS}

e-Consults are asynchronous clinician-to-clinician exchanges that rely on information in the patient's electronic health record (EHR). Requesting clinicians ask specific clinical questions; specialist physicians review the patient's EHR and document detailed recommendations as an EHR note (Fig. 1). Our institution, which first implemented e-consults as pilot programs in Cardiology and Dermatology, ${ }^{3}$ has completed over 35,000 e-consults in 50 subspecialty areas and extensively studied their use. ${ }^{4}$

Our primary outcome was e-consult proportion, defined as e-consult volume over total consult volume (e-consult volume plus traditional ambulatory consult volume). We used an interrupted time series (IST) model to assess the effect of the pandemic on e-consult proportion. We assessed daily volumes from February 1, 2020, through April 1, 2020; the defined date of "intervention" was March 11, 2020, when Massachusetts declared a COVID-19-related state of emergency. We included weekend/holiday as a covariate to account for differences in consult requests between business days and weekends. We excluded specialty areas with less than 20 e-consult requests, specialty areas that did not offer both an active e-consult and ambulatory consult option, and psychiatry as it included some requests for behavioral health resources without a need for specialist guidance.

This work, performed for administrative purposes, was exempt from review by the Partners Healthcare Institutional Review Board (IRB) per the IRB's policies. Statistical analysis was performed using SAS, version 9.4 (SAS Institute, Inc., Cary, NC).

\section{RESULTS}

Before March 11, 2020 ( $n=40$ days), a median of 565 ambulatory consults and $48 \mathrm{e}$-consults were requested daily. After March 11, 2020 ( $n=21$ days), a median of 144 ambulatory consults and 40 e-consults were requested daily. While both types of consult requests declined after March 11, the ambulatory consults declined more than the e-consults resulting in an increase in absolute e-consult proportion from 8.5 to $19.6 \%$. After adjusting for weekend and secular trend, we found e-consult proportion increased by 5\% (95\% CI 2-7\%) daily from pre-emergency declaration levels (Fig. 2).

\section{DISCUSSION}

We describe a significant increase in e-consult utilization relative to traditional ambulatory referrals following the COVID-19-related state of emergency declaration in Massachusetts. Our results suggest that e-consults can provide a mechanism for sustaining outpatient consultative care during this pandemic.

Study limitations include the fact that these results obtained from a single Boston-based academic medical center may not be generalizable, particularly to institutions without a strong econsult program in place. Additionally, this study design cannot assess the relative effectiveness of e-consults versus other types of virtual and in-person care delivery mechanisms.

These results suggest an increase in e-consult utilization associated with the COVID-19 pandemic in the USA. eConsults may be a promising method of ambulatory consultative care delivery as they can potentially replace some specialty consultations in a manner that provides clinical guidance while reducing the risk of in-person visits to both patients and physicians.

Prior Presentations This work has not previouslu been presented 
Referring clinician enters e-consult request in patient's EHR

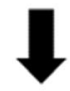

Request electronically transmitted to specialist physician

$\downarrow$

Specialist physician reviews e-consult request and related details in patient's EHR

$\downarrow$

Specialist documents recommendations as a note in patient's EHR

$\downarrow$

Referring clinician considers recommendations and determines next steps in care

Figure 1 e-Consult process. Referring clinicians enter a non-acute, focused clinical question into the patient's electronic health record (EHR). This question is electronically transmitted to a specialist physician who reviews the question and clinical information in the EHR and provides clinical guidance including recommendations for further diagnostic testing or therapeutic management via a note entered in the patient's EHR. The referring clinician reviews the specialist's recommendations and orders necessary testing or therapies.

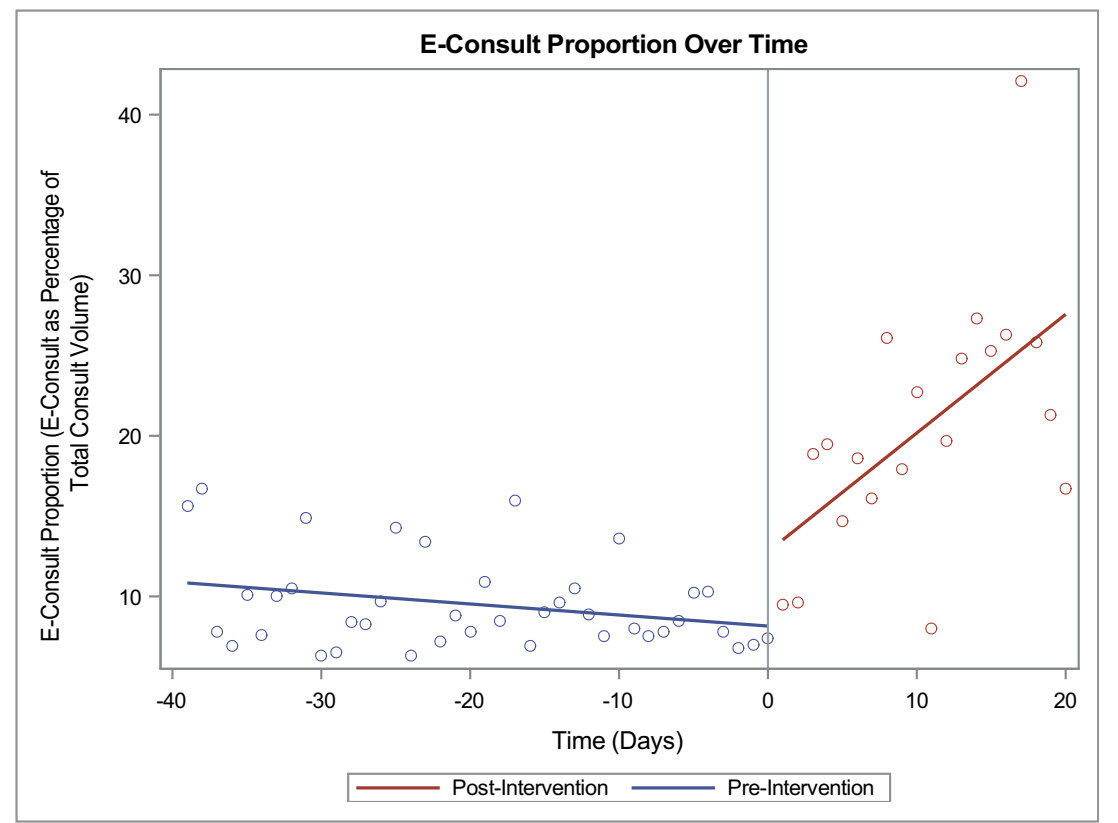

Figure 2 e-Consult proportion over time. This figure demonstrates e-consult proportion (the e-consult proportion of total referral volume) as a function of time. The vertical line at time 0 represents the date of intervention (March 11, 2020). Points to the left of this line represent data obtained prior to this date; points to the right represent data obtained after this date. Tick marks refer to the number of days in either direction. A linear model was fitted to the pre- and post-intervention data to better demonstrate the changing trend in e-consult proportion over time. 
Acknowledgments: The authors acknowledge Elizabeth Fonseca, SM, Program Director for Population Health Management, and Timothy G. Ferris, MD, MPH, Chief Executive Officer of the Massachusetts General Physicians Organization, for their support of electronic consults and other population health management efforts at Massachusetts General Hospital.

\section{Neelam A. Phadke, MD}

Division of Rheumatology, Allergy, and Immunology, Department of Medicine, Massachusetts General Hospital,

55 Fruit Street / Cox 201, Boston, MA 02114, USA

\section{Marcela G. del Carmen, MD, MPH}

Division of Gynecologic Oncology, Department of Obstetrics, Gynecology, and Reproductive Biology, Massachusetts General Hospital,

Boston, MA, USA

Susan A. Goldstein, MS

Jacqueline Vagle, MPH

Michael K. Hidrue, $\mathrm{PhD}$

Eirian Siegal Botti, BA

Performance Analysis and Improvement Unit, Massachusetts General Hospital,

Boston, MA, USA

\section{Jason H. Wasfy, MD, MPhil}

Cardiology Division, Department of Medicine, Massachusetts General Hospital,

Boston, MA, USA

Corresponding Author: Neelam A. Phadke, MD; Division of Rheumatology, Allergy, and Immunology, Department of Medicine,
Massachusetts General Hospital 55 Fruit Street / Cox 201, Boston, MA 02114, USA (e-mail: nphadke@partners.org).

Funding Information e-Consults are funded internally by the Massachusetts General Physicians Organization. No additional funding was obtained for this work.

\section{Compliance with Ethical Standards:}

Conflict of Interest: N.A.P. reports spousal employment by Chiesi Farmaceutici. J.H.W. reports consulting fees from Pfizer and Biotronik, career development awards from the American Heart Association (current), the National Institutes of Health (past), and Harvard Catalyst (past); honoraria from New England CEPAC for which he serves as vice chair; and travel compensation from the American College of Cardiology, academic medical centers, and academic conferences. He has additionally participated as a member on a public-private partnership convened by the U.S. Department of Health and Human Services on cardiac bundled payments. None of these relationships for either of these authors influenced the work presented here. The other authors disclose no conflicts of interest.

\section{REFERENCES}

1. World Health Organization. WHO characterizes COVID-19 as a pandemic. 11 Mar 2020. Accessed 18 Mar 2020. https://www.who.int/emergencies/ diseases/novel-coronavirus-2019/events-as-they-happen.

2. Lurie N, Carr BG. The role of telehealth in the medical response to disasters. JAMA Intern Med. 2018;178(6):745-6.

3. Wasfy JH, Rao SK, Chittle MD, Gallen KM, Isselbacher EM, Ferris TG. Initial results of a cardiac e-consult pilot program. J Am Coll Cardiol. 2014;44(24):2706-7.

4. Phadke NA, Wolfson AR, Mancini C, et al. Electronic consultations in Allergy/Immunology. J Allergy Clin Immunol Prac. 2019;7(8):2594-2602.

Publisher's Note: Springer Nature remains neutral with regard to jurisdictional claims in published maps and institutional affiliations. 